\title{
Continuous dependence of solutions to double dispersive equation with dissipative term
}

\author{
Mesude Elif Uysal* (D), Şevket Gür (D) \\ Sakarya University, Department of Mathematics, Sakarya, Turkey
}

\begin{abstract}
This work is focused on the properties of solutions to the initial-boundary value problem of the double dispersive-dissipative equation. It is established that the solution depends continuously on the dispersive and dissipative coefficients.
\end{abstract}

Mathematics Subject Classification (2020). 35B30, 35B35, 35B40, 35B45

Keywords. structural stability, double dispersive equations, continuous dependence

\section{Introduction}

Throughout this paper, $\Omega \subset \mathbb{R}^{n}$ is a bounded domain with sufficiently smooth boundary $\partial \Omega$. We study the following initial boundary value problem for nonlinear hyperbolic equations containing fourth order dissipative term:

$$
\begin{gathered}
u_{t t}-\Delta u-a \Delta u_{t t}+b \Delta^{2} u-d \Delta u_{t}+g\left(u_{t}\right)=f(u) \quad(x, t) \in \Omega \times[0, T], \\
u(x, 0)=u_{0}(x), \quad u_{t}(x, 0)=u_{1}(x) \quad x \in \Omega \subset \mathbb{R}^{n}(n \geqslant 3), \\
u=\Delta u \equiv 0 \quad(x, t) \in \partial \Omega \times[0, T], T>0,
\end{gathered}
$$

where $\Delta=\sum_{i=1}^{n} \frac{\partial^{2}}{\partial x_{i}^{2}}$ is an $n$-dimensional Laplace operator, $\Delta^{2}$ denotes biharmonic operator, and $g\left(u_{t}\right)=\alpha\left|u_{t}\right|^{m-2} u_{t}, f(u)=-\beta|u|^{p-2} u$. For $n=1,2$, one has $2<m, p \leqslant \infty$, but we consider here $n \geqslant 3$, so we have $2<m, p \leqslant \frac{2 n-2}{n-2}$. Because certain unknown parameters in the mathematical model are usually the results of an experimental work, they are actual physical parameters. In this equation, $a>0$ and $b>0$ are dispersive coefficients, $d>0$ is a dissipative coefficient expressesing energy loss in (1.1). There exist many examples of physical problems modeled by (1.1). For instance, the following equation is derived by the longitudinal displacement $u(x, t)$ of the elastic rod, in case of accepting the likelihood of energy exchange over the sides of the waveguide in the physical study of nonlinear wave propagation in waveguide;

$$
u_{t t}-u_{x x}=\frac{1}{4}\left(6 u^{2}-b u_{x x}+a u_{t t}\right)_{x x},
$$

\footnotetext{
*Corresponding Author.

Email addresses: elifuysal13@gmail.com (M. E. Uysal), sgur@sakarya@edu.tr (Ş. Gür)

Received: 10.07.2019; Accepted: 17.10.2020
} 
which is derived by Hamiltonian principle $[14,15]$. Likewise, the cubic double dispersive equation (CDDE) in general

$$
u_{t t}-u_{x x}=\frac{1}{4}\left(c u^{3}+6 u^{2}+a u_{t t}-b u_{x x}+d u_{t}\right)_{x x}
$$

can be achieved for $a, b, c, d>0[14,15]$. In [6], the authors take into account the nonlinear function $g\left(u_{t}\right)=a\left|u_{t}\right|^{m-2} u_{t}$ and the source term $f(u)=-b|u|^{p-2} u$ in (1.1) under the initial condition and homogeneous Dirichlet and Neumann boundary conditions in the region $\Omega \times(0, \infty)$. They used the Galerkion method to demonstrate the existence of a global weak solution to this problem. In [9], taking $f(u)=u^{2}$ and $g\left(u_{t}\right)=0$ in (1.1) and using Lie symmetries, invariant solutions are obtained to the following problem

$$
u_{t t}-c^{2} u_{x x}=\left(u^{2}+a u_{x x}+b u_{t t}\right)_{x x}+\mu u_{x x t} .
$$

In [18], the global existence of the weak solution to the problem

$$
\begin{gathered}
u_{t t}-\Delta u-\Delta u_{t t}+\Delta^{2} u+k \Delta u_{t}=\Delta f(u), \quad x \in \mathbb{R}^{n}, t>0, \\
u(x, 0)=u_{0}(x), \quad u_{t}(x, 0)=u_{1}(x),
\end{gathered}
$$

where $a=b=1$ in (1.1) has examined under conditions related to nonlinear term and initial data. In [16], the existence and uniqueness of a global solution for the Cauchy problem in the following equation have studied:

$$
\begin{gathered}
u_{t t}-u_{x x}-u_{x x t t}+u_{x x x x}-\alpha u_{x x t}=g(u)_{x x}, \\
u(x, 0)=u_{0}(x), \quad u_{t}(x, 0)=u_{1}(x) .
\end{gathered}
$$

On the other hand, the existence of a global solution of the following IBVP is established

$$
\begin{gathered}
u_{t t}-u_{x x}-a u_{x x t t}+b u_{x x x x}-d u_{x x t}=f(u)_{x x}, \\
u(0, t)=u(0, l)=0, \quad u_{x x}(0, l)=u_{x x}(l, t)=0, \quad t \geq 0 \\
u(x, 0)=u_{0}(x), \quad u_{t}(x, 0)=u_{1}(x), \quad x \in \bar{\Omega},
\end{gathered}
$$

where $\Omega=(0, l)$ [3]. In [8], blow-up phenomena of generalized double dispersion equations

$$
u_{t t}-u_{x x}-u_{x x t}+u_{x x x x}-u_{x x t t}=f\left(u_{x}\right)_{x}
$$

is surveyed. The authors establish a blow-up result for the solutions with arbitrary high initial energy, and give some upper bounds for blow-up time $T^{*}$, depending on sign and size of initial energy. In [7], the existence and nonexistence of global weak solutions to the Cauchy problem for a higher order generalized Boussinesq-type equation with hydrodynamical damped term in $n$-dimensional space is considered. It can be seen from previous studies that the problem (1.1)-(1.3) is solved in the proper spaces and sufficient conditions for existence and uniqueness of the solution to the problem are derived. In the current paper, we investigate the continuous dependence of the solution of (1.1)-(1.3) on the coefficients of that equation in $L^{2}(\Omega)$ space. These coefficients are usually determined via conditions of the experiments that are subject to variability and chance. Modeling physical phenomena mathematically with appropriate parameters determined from experiments, one tries to predict the outcome of certain physical processes [17]. With these difficulties in mind, one must raise the question that if the mathematical model is derived correctly? That is, do small changes of the set of parameters only result in small variations of the outcome? To answer this question, this paper aims at surveying how the results are affected when the small changes occur in parameters $a, d, \alpha$, and $\beta$. Recently, many important works have been done on deriving estimates about structural stability of the equation. Those of works are examined for multidimensional equations in following main books $[1,2]$, the articles $[4,5,10-13,19]$, and references therein.

Definition 1.1. We call a weak solution of the problem a function $u \in L^{\infty}\left(0, T ; H_{0}^{2}(\Omega) \cap\right.$ $\left.L^{p}(\Omega)\right), u_{t} \in L^{\infty}\left(0, T ; H_{0}^{1}(\Omega) \cap L^{m}\left(Q_{T}\right)\right)$, satisfying (1.1) in the sense of distributions [7]. 
Remark 1.2. The interested reader is referred to Theorem 3.1. in [7] for the proof of the existence and uniqueness of a weak solution for the problem (1.1) - (1.3).

\section{A priori estimates}

Theorem 2.1. Let $\left(u_{0}, u_{1}\right)$ belong to $H^{2}(\Omega) \cap H_{0}^{1}(\Omega) \times H_{0}^{1}(\Omega)$. Then the solution $u$ of the problem (1.1)-(1.3) satisfies the following inequalities:

$$
\left\|u_{t}(t)\right\|^{2} \leqslant A_{1},\|\nabla u(t)\|^{2} \leqslant A_{1},\|\Delta u(t)\|^{2} \leqslant A_{2},\left\|\nabla u_{t}(t)\right\|^{2} \leqslant A_{3},
$$

where $A_{1}, A_{2}, A_{3}>0$ depend on the coefficients of the equation (1.1) and the initial data.

Proof. Multiplying (1.1) by $u_{t}$ in $L^{2}(\Omega)$, we get

$$
\begin{aligned}
\frac{d}{d t}\left[\frac{1}{2}\left\|u_{t}(t)\right\|^{2}+\frac{1}{2}\|\nabla u(t)\|^{2}+\frac{b}{2}\|\Delta u(t)\|^{2}\right. & \left.+\frac{a}{2}\left\|\nabla u_{t}(t)\right\|^{2}+\frac{\beta}{2}\|u(t)\|_{p}^{p}\right] \\
& +d\left\|\nabla u_{t}(t)\right\|^{2}+\alpha\left\|u_{t}(t)\right\|_{m}^{m}=0 .
\end{aligned}
$$

It follows from (2.2) that

$$
E_{u}(t):=\frac{1}{2}\left\|u_{t}(t)\right\|^{2}+\frac{1}{2}\|\nabla u(t)\|^{2}+\frac{b}{2}\|\Delta u(t)\|^{2}+\frac{a}{2}\left\|\nabla u_{t}(t)\right\|^{2}+\frac{\beta}{2}\|u(t)\|_{p}^{p} \leqslant E_{u}(0) .
$$

Hence, all of the estimates in (2.1) follow from (2.3).

Theorem 2.2. Suppose that $u_{0} \in L^{2}(\Omega)$, and $u_{1} \in H^{2}(\Omega) \cap H_{0}^{1}(\Omega)$. Then the solution of the problem (1.1)-(1.3) satisfies the following regular estimates:

$$
\left\|\nabla u_{t t}(t)\right\|^{2} \leqslant A_{4} \quad \forall t \in[0, T]
$$

where $A_{4}$ depends on the parameters of the system and initial data.

Proof. To get uniform estimate, (1.1) is differentiated with respect to $t$ :

$$
u_{t t t}-\Delta u_{t}-d \Delta u_{t t}+b \Delta^{2} u_{t}-a \Delta u_{t t t}+\alpha(m-1)\left|u_{t}\right|^{m-2} u_{t t}+\beta(p-1)|u|^{p-2} u_{t}=0 .
$$

Multiplying $(2.5)$ by $u_{t t}$ in $L^{2}(\Omega)$ gives

$$
\begin{aligned}
\frac{d}{d t}\left[\frac{1}{2}\left\|u_{t t}(t)\right\|^{2}\right. & \left.+\frac{1}{2}\left\|\nabla u_{t}(t)\right\|^{2}+\frac{b}{2}\left\|\Delta u_{t}(t)\right\|^{2}+\frac{a}{2}\left\|\nabla u_{t t}(t)\right\|^{2}\right] \\
& +d\left\|\nabla u_{t t}(t)\right\|^{2}+\alpha(m-1) \int_{\Omega}\left|u_{t}\right|^{m-2} u_{t t}^{2} d x+\beta(p-1) \int_{\Omega}|u|^{p-2} u_{t} u_{t t} d x=0 .
\end{aligned}
$$

We estimate last two integral terms on the left hand side of the equation. First, we have

$$
\begin{aligned}
\alpha(m-1) \int_{\Omega}\left|u_{t}\right|^{m-2}\left|u_{t t}\right|^{2} d x & \leqslant \alpha(m-1) \max \left|u_{t}\right|^{m-2}|\Omega| \int_{\Omega}\left|u_{t t}\right|^{2} d x \\
& \leqslant \alpha(m-1) 2|\Omega| E_{u}(0)^{\frac{m-2}{2}}\left\|u_{t t}\right\|^{2} \\
& \leqslant C\left\|u_{t t}(t)\right\|^{2} \\
& \leqslant C_{1}\left\|\nabla u_{t t}(t)\right\|^{2},
\end{aligned}
$$

where $C_{1}=2 C \alpha(m-1) E_{u}(0)^{\frac{m-2}{2}}|\Omega|$. For the last term, we get

$$
\begin{aligned}
\beta(p-1) \int_{\Omega}|u|^{p-2} u_{t} u_{t t} d x & \leq \beta(p-1) \max |u|^{p-2}\left\|u_{t}(t)\right\|\left\|u_{t t}(t)\right\| \\
& \leqslant \beta(p-1) C(\Omega)\|\nabla u(t)\|^{p-2}\left\|u_{t}(t)\right\|\left\|u_{t t}(t)\right\| \\
& \leqslant C_{2}\left[\left\|\nabla u_{t}(t)\right\|^{2}+\left\|\nabla u_{t t}(t)\right\|^{2}\right]
\end{aligned}
$$


where $C_{2}=\frac{\beta(p-1) C(\Omega) A_{1}^{\frac{p-2}{2}}}{2}$. Combining these in (2.6), and simplifying the inequality, we have

$$
\frac{d}{d t} E_{u_{t}}(t) \leqslant C_{2}\left\|\nabla u_{t}(t)\right\|^{2}+\left(C_{1}+C_{2}\right)\left\|\nabla u_{t t}(t)\right\|^{2}
$$

where $E_{u_{t}}(t)=\frac{1}{2}\left\|u_{t t}(t)\right\|^{2}+\frac{1}{2}\left\|\nabla u_{t}(t)\right\|^{2}+\frac{b}{2}\left\|\Delta u_{t}(t)\right\|^{2}+\frac{a}{2}\left\|\nabla u_{t t}(t)\right\|^{2}$. Therefore, we obtain

$$
\frac{d}{d t} E_{u_{t}}(t) \leqslant M_{1} E_{u_{t}}(t)
$$

where $M_{1}=\max \left\{\frac{1}{2}, \frac{a}{2}, C_{1}+C_{2}\right\}$. Integrating the last inequality, we get

$$
E_{u_{t}}(t) \leqslant e^{M_{1} t} E_{u_{t}}(0)
$$

Hence, we have

$$
\left\|\nabla u_{t t}(t)\right\|^{2} \leqslant \frac{2 E_{u_{t}}(0)}{a} e^{M_{1} t}
$$

which yields (2.4) with $A_{4}=\frac{2 E_{u_{t}}(0)}{a} e^{M_{1} t} \forall t \in[0, T]$.

\section{Continuous dependence on all parameters}

In general, the subject of the continuous dependence is examined on coefficients separately. However, in this section, we show that the solution of the problem (1.1)-(1.3) depends continuously on coefficients $a, d, \alpha, \beta$ under the same inequality.

Suppose that $u$ is the solution of

$$
\begin{gathered}
u_{t t}-\Delta u-a_{1} \Delta u_{t t}+b \Delta^{2} u-d_{1} \Delta u_{t}+\alpha_{1}\left|u_{t}\right|^{m-2} u_{t}+\beta_{1}|u|^{p-2} u=0, \\
u(x, 0)=u_{0}(x), \quad u_{t}(x, 0)=u_{1}(x) \quad x \in \Omega \subset \mathbb{R}^{n}(n \geqslant 3) \\
u=\Delta u \equiv 0 \quad(x, t) \in \partial \Omega \times[0, T], T>0,
\end{gathered}
$$

and $v$ is the solution of the following IBVP:

$$
\begin{gathered}
v_{t t}-\Delta v-a_{2} \Delta v_{t t}+b \Delta^{2} v-d_{2} \Delta v_{t}+\alpha_{2}\left|v_{t}\right|^{m-2} v_{t}+\beta_{2}|v|^{p-2} v=0, \\
v(x, 0)=u_{0}(x), \quad v_{t}(x, 0)=u_{1}(x) \quad x \in \Omega \subset \mathbb{R}^{n}(n \geqslant 3), \\
v=\Delta v \equiv 0 \quad(x, t) \in \partial \Omega \times[0, T], T>0 .
\end{gathered}
$$

Therefore $w$ is a solution of the following IBVP:

$$
\begin{gathered}
w_{t t}-\Delta w-a_{1} \Delta w_{t t}-a \Delta v_{t t}+b \Delta^{2} w-d_{1} \Delta w_{t}-d \Delta v_{t} \\
+\alpha_{1}\left(\left|u_{t}\right|^{m-2} u_{t}-\left|v_{t}\right|^{m-2} v_{t}\right)+\alpha\left|v_{t}\right|^{m-2} v_{t} \\
+\beta_{1}\left(|u|^{p-2} u-|v|^{p-2} v\right)+\beta|v|^{p-2} v=0, \\
w(x, 0)=0, \quad w_{t}(x, 0)=0 \quad x \in \Omega \subset \mathbb{R}^{n}, \\
w=\Delta w \equiv 0 \quad(x, t) \in \partial \Omega \times[0, T], T>0,
\end{gathered}
$$

where $w=u-v, a=a_{1}-a_{2}, \beta=\beta_{1}-\beta_{2}, d=d_{1}-d_{2}, \alpha=\alpha_{1}-\alpha_{2}$.

Theorem 3.1. The solution $w$ of the problem (3.7)-(3.9) satisfies the inequality

$$
\left\|\nabla w_{t}(t)\right\|^{2}+\frac{b}{2}\|\Delta w(t)\|^{2} \leqslant\left\{\frac{A_{4}}{2} a^{2}+\frac{A_{3}}{2} d^{2}+\left(d_{0}^{m-1} A_{3}^{\frac{m-1}{2}}\right) \alpha^{2}+\left(d_{1}^{p-2} A_{1}^{\frac{p-1}{2}}\right) \beta^{2}\right\} t e^{M_{2} t},
$$

where $A_{1}, A_{3}, A_{4}, M_{2} \geq 0$ depend on the coefficients of the equation (1.1) and the initial data. 
Proof. First, multiplying (3.7) by $w_{t}$ we get

$$
\begin{aligned}
\frac{d}{d t} & {\left[\frac{1}{2}\left\|w_{t}(t)\right\|^{2}+\frac{1}{2}\|\nabla w(t)\|^{2}+\frac{a_{1}}{2}\left\|\nabla w_{t}(t)\right\|^{2}+\frac{b}{2}\|\Delta w(t)\|^{2}\right] } \\
& +a\left(\nabla w_{t}, \nabla v_{t t}\right)+d_{1}\left\|\nabla w_{t}(t)\right\|^{2}+d\left(\nabla w_{t}, \nabla v_{t}\right) \\
& +\alpha_{1}\left(\left|u_{t}\right|^{m-2} u_{t}-\left|v_{t}\right|^{m-2} v_{t}, w_{t}\right)+\alpha\left(\left|v_{t}\right|^{m-2} v_{t}, w_{t}\right) \\
& +\beta_{1}\left(|u|^{p-2} u-|v|^{p-2} v, w_{t}\right)+\beta\left(|v|^{p-2} v, w_{t}\right)=0 .
\end{aligned}
$$

Rearranging (3.10), we have

$$
\begin{aligned}
& \frac{d}{d t}\left[\frac{1}{2}\left\|w_{t}(t)\right\|^{2}+\frac{1}{2}\|\nabla w(t)\|^{2}+\frac{a_{1}}{2}\left\|\nabla w_{t}(t)\right\|^{2}+\frac{b}{2}\|\Delta w(t)\|^{2}\right]+d_{1}\left\|\nabla w_{t}(t)\right\|^{2} \\
& \quad+\alpha_{1}\left(\left|u_{t}\right|^{m-2} u_{t}-\left|v_{t}\right|^{m-2} v_{t}, w_{t}\right) \leqslant\left|a\left(\nabla w_{t}, \nabla v_{t t}\right)\right|+\left|d\left(\nabla w_{t}, \nabla v_{t}\right)\right| \\
& \quad+\left|\alpha\left(\left|v_{t}\right|^{m-2} v_{t}, w_{t}\right)\right|+\left|\beta_{1}\left(|u|^{p-2} u-|v|^{p-2} v, w_{t}\right)\right|+\left|\beta\left(|v|^{p-2} v, w_{t}\right)\right| .
\end{aligned}
$$

Now, we get the upper boundaries for the right hand side of (3.11). Due to Cauchy-Schwarz inequality, we have

$$
\left|a\left(\nabla w_{t}, \nabla v_{t t}\right)\right| \leqslant \frac{a^{2}\left\|\nabla v_{t t}\right\|^{2}}{2}+\frac{\left\|\nabla w_{t}(t)\right\|^{2}}{2},
$$

and

$$
\left|d\left(\nabla w_{t}, \nabla v_{t}\right)\right| \leqslant \frac{d^{2}\left\|\nabla v_{t}(t)\right\|^{2}}{2}+\frac{\left\|\nabla w_{t}(t)\right\|^{2}}{2} .
$$

Thanks to $\varepsilon$-Young and Sobolev inequalities, with $m, p \leqslant \frac{2 n-2}{n-2}$, we obtain

$$
\begin{aligned}
\left|\left(\left|v_{t}\right|^{m-2} v_{t}, w_{t}\right)\right| & \leqslant \int\left|v_{t}\right|^{m-2}\left|v_{t}\right|\left|w_{t}\right| d x \\
& \leqslant\left\|v_{t}(t)\right\|_{2(m-1)}^{m-1}\left\|w_{t}(t)\right\| \\
& \leqslant d_{0}^{m-1}\left\|\nabla v_{t}(t)\right\|^{m-1}\left\|w_{t}(t)\right\| \\
& \leqslant \alpha d_{0}^{m-1} A_{3}^{\frac{m-1}{2}}+\frac{1}{4 \alpha}\left\|w_{t}(t)\right\|^{2} .
\end{aligned}
$$

Similarly, we have

$$
\left|\left(|v|^{p-2} v, w_{t}\right)\right| \leqslant \beta d_{1}^{p-2} A_{1}^{\frac{p-1}{2}}+\frac{1}{4 \beta}\left\|w_{t}(t)\right\|^{2} .
$$

Besides, it can be easily shown that $\int_{\Omega}\left(\left|u_{t}\right|^{m-2} u_{t}-\left|v_{t}\right|^{m-2} v_{t}\right) w_{t} d x \geqslant 0$.

Moreover, using the mean value theorem, Hölder's and Sobolev inequalities with $p \leqslant \frac{2 n-2}{n-2}$ and $\varepsilon$-Young inequality with $\varepsilon=\beta_{1}$, we have

$$
\begin{aligned}
\left|\int_{\Omega}\left(|u|^{p-2} u-|v|^{p-2} v\right) w_{t} d x\right| & \leqslant(p-1) \int_{\Omega}\left|w \| w_{t}\right|\left(|u|^{p-2}+|v|^{p-2}\right) d x \\
& \leqslant(p-1)\|w(t)\|_{\frac{2 n}{n-2}}\left\|w_{t}(t)\right\|\left(\|u(t)\|_{(p-2) n}^{p-2}+\|v(t)\|_{(p-2) n}^{p-2}\right) \\
& \leqslant(p-1)\left\|w_{t}(t)\right\| C_{1}\|\nabla w(t)\| C_{2}\left(\|\nabla u(t)\|^{p-2}+\|\nabla v(t)\|^{p-2}\right) \\
& \leqslant(p-1) C_{1} C_{2} 2 A_{1}^{\frac{p-2}{2}}\left\|w_{t}(t)\right\|\|\nabla w(t)\| \\
& \leqslant C_{3}\left\|w_{t}(t)\right\|\|\nabla w(t)\| \\
& \leqslant \beta_{1} C_{3}^{2}\|\nabla w(t)\|^{2}+\frac{1}{4 \beta_{1}}\left\|w_{t}(t)\right\|^{2} .
\end{aligned}
$$


Combining all boundaries for the right hand side of (3.11), we get

$$
\frac{d}{d t} E_{w}(t) \leqslant M_{2} E_{w}(t)+B
$$

where

$$
M_{2}=\max \left\{\beta_{1}^{2} C_{3}^{2}, a_{1} / 2,1+d_{1}\right\}
$$

and

$$
B=\frac{a^{2}}{2} A_{4}+\frac{d^{2}}{2} A_{3}+\alpha^{2} d_{0}^{m-1} A_{3}^{\frac{m-1}{2}}+\beta^{2} d_{1}^{p-2} A_{1}^{\frac{p-1}{2}} .
$$

Solving differential inequality (3.12), we obtain

$$
E_{w}(t) \leqslant B t e^{M_{2} t}
$$

It is proved that if $a \rightarrow 0, d \rightarrow 0, \alpha \rightarrow 0, \beta \rightarrow 0$ at the same time, then $w \rightarrow 0, \forall t \in[0, T]$, which is the desired result.

\section{References}

[1] K.A. Ames and B. Straughan, Non-Standard and improperly posed problems, Mathematics in Science and Engineering Series, Academic Press, New York, 194, 1997.

[2] N. Bellomo and L. Preziosi, Modelling mathematical methods and scientific computation, CRC, Boca Raton, 1995.

[3] G. Chen, Y. Wang and S. Wang, Initial boundary value problem of the generalized cubic double dispersion equation, J. Math. Anal. Appl. 299 (2), 563-577, 2004.

[4] A.O. Çelebi, Ş. Gür and V.K. Kalantarov, Structural stability and decay estimate for marine riser equations, Math. Comput. Model. 54 (11-12), 3182-3188, 2011.

[5] A.O. Çelebi and V.K. Kalantarov, Decay of solutions and structural stability for the coupled Kuramoto-Sivashinsky-Ginzburg-Landau equations, Appl. Anal. 94 (11), 23422354, 2015.

[6] H.F. Di and Y.D. Shang, Global existence and asymptotic behavior of solutions for the double dispersive-dissipative wave equation with nonlinear damping and source terms, Bound. Value Probl. 2015, Article No: 29, 2015.

[7] H.F. Di and Y.D. Shang, Cauchy Problem for a higher order generalized Boussinesqtype equation with hydrodynamical damped term, Appl. Anal. 95 (3), 690-714, 2016.

[8] H.F. Di and Y.D. Shang, Blow-Up Phenomena for a class of Generalized Double Dispersive Equations, Acta Math. Acad. Sci. 39 (2), 567-579, 2019.

[9] V.V. Gursky and A.M. Samsonov, Symmetries and exact solutions to a nonlinear doubly dispersive equation with dissipation, Conference: Day on Diffraction 2001, Proceedings, International Seminar, 2001.

[10] Ş. Gür and I. Güleç, Continuous dependence of solutions to fourth-order nonlinear wave equation, Hacet. J. Math Stat. 45, 367-371, 2016.

[11] Ş. Gür and M.E. Uysal, Continuous dependence of solutions to strongly damped nonlinear Klein-Gordon Equation, Turkish J. Math. 42, 904-910, 2018.

[12] C. Lin and L.E. Payne, Continuous dependence on the Soret coefficient for double diffusive convection in Darcy flow, J. Math. Anal. Appl. 342, 311-325, 2008.

[13] L.E. Payne and B. Straughan, Convergence and continuous Dependence for the Brinkman-Forchheimer equations, Stud. Appl. Math. 102, 419-439, 1999.

[14] A.M. Samsonov, Nonlinear Strain Waves in Elastic Waveguides, in: Jeffrey A., Engelbrecht J. (eds) Nonlinear Waves in Solids. CISM Courses and Lectures (International Centre for Mechanical Sciences), 341, Springer, Vienna, 1994.

[15] A.M. Samsonov and E.V. Sokurinskaya, Energy Exchange Between Nonlinear Waves in Elastic Waveguides and External Media, Nonlinear Waves in Active Media, Research Reports in Physics, Springer, Heidelberg, 1989. 
[16] S. Wang and G. Chen, Cauchy problem of the generalized double dispersion equation, Nonlinear Anal. 64 (1) 159-173, 2006.

[17] M. Waurick, On the continuous dependence on the coefficients of evolutionary equations, Habilitation thesis, TU Dresden, arXiv: 1606.07731, 2016.

[18] R. Xu, Y. Liu and T. Yu, Global existence of solution for Cauchy problem of multidimensional generalized double dispersion equations, Nonlinear Anal. 71 (10), 49774983, 2009.

[19] M. Yaman and Ş. Gür, Continuous dependence for the damped nonlinear hyperbolic equation, Math. Comput. Appl. 16 (2), 437-442, 2011. 